\title{
Temperature and Humidity Forecast via Univariate Partial Least Square and Principal Component Analysis
}

\author{
Sutikno $^{1 a^{*},}$ Zahrotun Nisaa'1b, Kartika Nur 'Anisa'1c \\ ${ }^{1}$ Department of Statistics, Faculty of Mathematics Computing and Data Science, Institut Teknologi Sepuluh Nopember, \\ Kampus Sukolilo, Surabaya 60111, INDONESIA. E-mail: sutikno@statistika.its.ac.id ${ }^{a}$; zahrotunnisaa11@ gmail.com; \\ kartika.nuranisa9@gmail.com ${ }^{\mathrm{c}}$ \\ * Corresponding Author: sutikno@statistika.its.ac.id ${ }^{\mathrm{a}}$ \\ Received: $21^{\text {st }}$ April $2019 \quad$ Revised : 6 $6^{\text {th }}$ August $2019 \quad$ Published: $30^{\text {th }}$ September 2019 \\ DOI : https://doi.org/10.22452/mjs.sp2019no2.1
}

\begin{abstract}
Indonesian Meteorology, Climatology, and Geophysics Agency (BMKG) uses Numerical Weather Prediction (NWP) for short-term weather forecast but it gives biased result. Therefore, this study implements Univariate Partial Least Square (PLS) as Model Output Statistics (MOS) for temperature and humidity forecast. This study uses the maximum temperature (Tmax), minimum temperature (Tmin), and relative humidity $(\mathrm{RH})$ which are called response variables and NWP as predictor variable. The results show that the performance of the model based on Root Mean Square Error of Prediction (RMSEP) are considered to be good and intermediate. The RMSEP for Tmax in all stations is intermediate (0.9-1.2), Tmin in three stations is good (0.5-0.8), and humidity in three stations is also good (2.6-5.0). The prediction result from the PLS is more accurate than the NWP model and able to correct an $89.94 \%$ of the biased NWP for Tmin forecasting.
\end{abstract}

Keywords: MOS, NWP, PCA, PLS, Temperature and Humidity.

\section{INTRODUCTION}

Indonesia is one of the archipelago states with a tropical climate, having a dynamic and complex weather and atmospheric system. The atmosphere also has a significant role in the global weather and climate systems (Tjasyono, 2004). Weather is considered to be the part that cannot be separated from human activity and influences the various areas of life. Dealing with it, an efficient method is needed for weather forecasting, especially in the shortterm forecasting (Wardani, 2010). Indonesian Meteorology, Climatology, and Geophysics Agency (BMKG) has forecasted a short-term weather by comparing and observing a weather pattern and condition that happened the day before, and generally, the accuracy of forecasting will vary since it largely depends on the forecaster's experience.

Information about weather forecasts has been published by BMKG including maximum temperature (Tmax), minimum temperature (Tmin), and the relative humidity (RH). Since 2004, BMKG has been doing a study for a short-term weather forecasting using Numerical Weather Prediction (NWP) data, but the result of the NWP forecasting was biased for a location that had complex highresolution topography and vegetation. Thus, Clark et al. (2001) used the Model Output Statistics (MOS) to optimize the utilization of NWP output to produce more accurate weather forecasts.

MOS is a method for modeling of the relation between the weather observation result and the NWP output based on a regression method. MOS will determine the statistical relationship between the predictor variable and the NWP model response variable for a certain time projection (Glahn and Lowry, 1972). In this study, we use Univariate Partial Least 
Square (PLS) as the MOS method, PLS utilizes a univariate response and only has a single objective function and a single response variable.

The response variable is weather observation data, while the predictor variable is the output data of the Numerical Weather Prediction Conformal Cubic Atmospheric Model (NWP CCAM). The NWP data is taken from 9 measurement grids for every variable so that the complexity will be high and the multicollinearity potentially occurs. This high complexity can be tolerated using PCA (Principal Component Analysis) process to reduce the dimension of the grid. The result from this dimension reduction will be used as the predictor variable for the PLS. Then, the PLS result through the PCA as its preprocessing stage will be compared with the actual data and the NWP model by looking at RMSEP (Root-Mean-Square-Error Prediction) and \%IM (percentage improval) criteria.

We describe the Principal Component
Analysis (PCA) method, MOS Modeling using PLS, variables used, and model evaluation in section 2. In section 3, we apply the method to forecast temperature and humidity, also show the results of our analysis. Finally, section 4 presents the conclusion of this study. In this study, we use statistical approach to explain about temperature and humidity forecast.

\section{METHODS AND MATERIALS}

\subsection{Principal Component Analysis}

Principal component analysis (PCA) is to reduce multicollinearity and the dimension of data. The result will be a new data with reduced variable but still able to explain the variability of data (Joliffe, 1986). If a random Vector $\mathbf{X}^{T}=\left[X_{1}, X_{2}, \ldots, X_{p}\right]$ has a covariance matrix of $\boldsymbol{\Sigma}$ with the eigenvalue of $\lambda_{1} \geq \lambda_{2} \geq \ldots \geq \lambda_{p} \geq 0$, then the linear combination will be in (1).

$$
\begin{aligned}
& P C_{1}=\mathbf{e}_{1}^{T} \mathbf{X}=e_{11} X_{1}+e_{12} X_{2}+\ldots+e_{1 p} X_{p} \\
& \vdots \\
& P C_{p}=\mathbf{e}_{p}{ }^{\mathrm{T}} \mathbf{X}=e_{p 1} X_{1}+e_{p 2} X_{2}+\ldots+e_{p p} X_{p}
\end{aligned}
$$

$P C_{p}=$ the $p^{\text {th }}$ linear combination, the $p^{\text {th }}$ biggest variance

$X_{p}=$ the $p^{\text {th }}$ origin variable

$\mathbf{e}_{p}=$ the $p^{\text {th }}$ eigenvector

The $i^{\text {th }}$ linear combination can be generally written as follows in (2).

$$
P C_{i}=\mathbf{e}_{i}^{T} \mathbf{X}, i=1,2, \ldots, p
$$

So that, $\operatorname{Cov}\left(P C_{i}, \mathrm{PC}_{k}\right)=\mathbf{e}_{i}^{T} \boldsymbol{\Sigma} \mathbf{e}_{k}, i, k=1,2, \ldots, \mathrm{p}$. The principal components do not have any correlation among each of them and have the same variance with eigenvalue from $\Sigma$, so as in (3).

$$
\sigma_{11}+\sigma_{22}+\ldots+\sigma_{p p}=\sum_{i=1}^{p} \operatorname{Var}\left(X_{i}\right)=\lambda_{1}+\lambda_{2}+\ldots+\lambda_{p}=\sum_{i=1}^{p} \operatorname{Var}\left(P C_{i}\right)
$$

The number of principal components is $k$ where $k<p$ and the proportion of total variance that can be explained by the $k^{\text {th }}$ principal component as follows: 


$$
\text { Variance Proportion for } k^{\text {th }} P C=\frac{\lambda_{k}}{\lambda_{1}+\lambda_{2}+\ldots+\lambda_{p}} \quad \text {. }
$$

According to Johnson and Winchern (2007), there are several points to determine the amount of PC:

1. Observing the scree plot, as it shows the amount of eigenvalue $\lambda_{i}$. If the line created at the scree plot has a certain big range, then the PC on this line will be taken.

2. The amount of the PC taken is chosen according to the amount of eigenvalue that is greater than 1 (if the PC is obtained from the correlation matrix).
3. The amount of PC taken should have a cumulative variance percentage of $80 \%$ to $90 \%$. It means that the PC should be able to explain data variability of at least $80 \%$.

\subsection{MOS Modeling using PLS}

MOS is a modeling between the weather observation result and the output of NWP based on regression. According to Wilks (2006), the general mathematical model of MOS is shown in (5).

$$
\hat{Y}_{t}=f_{M O S}\left(X_{t}\right)
$$

$$
\begin{aligned}
& \hat{Y}_{t}=\text { weather forecast at the time- } t \\
& X_{t}=\text { output variables of NWP at the time- } t
\end{aligned}
$$

PLS (Partial Least Square) is an efficient statistical method for predicting a small data sample with a lot of variables that might be correlated with each other. By doing a computer calculation, PLS becomes easier to be implemented for a great amount of data without the need to provide assumption (Wilks, 2006). In PLS, the dimensional reduction and the regression process are done simultaneously. Then $\mathbf{T}$ is denoted as the latent variable or score, which is obtained from random sample variable matrix decomposition $n \times c$. $\mathbf{P}$ is called the $\mathrm{X}$-loadings $p \times c$ and $\mathbf{Q}$ is called Y-loadings $q \times c$. The PLS is based on the latent component decomposition from (6)

$$
\begin{aligned}
& \mathbf{X}=\mathbf{T} \mathbf{P}^{T}+\mathbf{E} \\
& \mathbf{Y}=\mathbf{T} \mathbf{Q}^{T}+\mathbf{F}
\end{aligned} .
$$

Hence, the $\mathbf{X}$ matrix is $n \times p$ and $\mathbf{Y}$ is $n \times q$. $\mathbf{E}$ and $\mathbf{F}$ are residual matrices that are each of which are $n \times p$ and $n \times q$.

The PLS is just like the principal component regression that is a method that forms the latent component matrix $\mathbf{T}$ as the linear transformation from $\mathbf{X}$,

$$
\mathbf{T}=\mathbf{X W}^{*}
$$

$\mathbf{W} *$ is the weighting matrix sized $p \times c$ with $c$ is the number of latent components. The $\mathbf{W} *$ can be obtained using (8).

$$
\mathbf{W}^{*}=\mathbf{W}\left(\mathbf{P}^{T} \mathbf{W}\right)^{-1}
$$


The latent component is used to predict $\mathbf{Y}$, substituting the origin variable, $\mathbf{X}$. When $\mathbf{T}$ is formed, we can then obtain $\mathbf{Q}^{T}$ from the smallest quadratic method as in (9).

$$
\hat{\mathbf{Q}}^{T}=\left(\mathbf{T}^{T} \mathbf{T}\right)^{-1} \mathbf{T}^{T} \mathbf{Y}
$$

From equation (6), $\mathbf{Y}=\mathbf{T} \mathbf{Q}^{T}+\mathbf{F}$ and the matrix $\mathbf{B}$ is a regression coefficient matrix for the model $\mathbf{Y}=\mathbf{X B}+\mathbf{F}$, then the equation (10) is obtained.

$$
\begin{aligned}
& \mathbf{X B}=\mathbf{T} \mathbf{Q}^{T} \\
& \mathbf{X B}=\mathbf{X W} * \mathbf{Q}^{T} \\
& \mathbf{B}=\mathbf{W} * \mathbf{Q}^{T}
\end{aligned}
$$

The estimator of $\mathbf{B}$ is $\hat{\mathbf{B}}=\mathbf{W} *\left(\mathbf{T}^{T} \mathbf{T}\right)^{-1} \mathbf{T}^{T} \mathbf{Y}$. So that we can obtain a conjecture for $\mathbf{Y}$ as in (11).

$$
\begin{aligned}
& \hat{\mathbf{Y}}=\mathbf{X} \hat{\mathbf{B}} \\
& \hat{\mathbf{Y}}=\left[\mathbf{T} \mathbf{W}^{-1} \mathbf{W}\left(\mathbf{T}^{T} \mathbf{T}\right)^{-1} \mathbf{T}^{T} \mathbf{Y}\right] \\
& \hat{\mathbf{Y}}=\left[\mathbf{T I}\left(\mathbf{T}^{T} \mathbf{T}\right)^{-1} \mathbf{T}^{T} \mathbf{Y}\right] \\
& \hat{\mathbf{Y}}=\left[\mathbf{T}\left(\mathbf{T}^{T} \mathbf{T}\right)^{-1} \mathbf{T}^{T} \mathbf{Y}\right]
\end{aligned}
$$

The PLS can be used for both univariate response and multivariate response. This study is utilizing the PLS for the univariate response with the intent to obtain each modeling result from the response variable separately. The amount of latent variable is determined by a statistic assessing the accuracy of estimation, Prediction Residual Sum of Square (PRESS). The PRESS value for the univariate response is shown in (12).

$$
\text { PRESS }=\sum_{t=1}^{n}\left(y_{t}-\hat{y}_{t-1}\right)^{2}
$$

The modeling using PLS is done when the response variable is to be analyzed separately so that $Y$ is a response matrix variable $n \times 1$. For a certain weight amount $\mathbf{w}_{\mathbf{i}}=\left(w_{11}, \ldots, w_{p i}\right)^{T}$, the covariance between the response variable $Y$ and the random variable $T_{i}=w_{1 i} X_{1}+w_{2 i} X_{2} \ldots+w_{p i} X_{p}$ can be obtained using (13)

$$
\operatorname{COV}\left(Y, T_{i}\right)=\frac{1}{n} \mathbf{w}_{i}^{T} \mathbf{X}^{T} \mathbf{Y}
$$

Covariance between $T_{i}$ and $T_{j}$ for $i \neq j ; j=1,2, \ldots, c$

$$
\operatorname{COV}\left(Y, T_{i}\right)=\frac{1}{n} \mathbf{w}_{i}^{T} \mathbf{X}^{T} \mathbf{w}_{j}=\frac{1}{n} \mathbf{t}_{i}^{T} \mathbf{t}_{j}
$$


$\mathbf{W}$ is defined to be the square of the covariance between $Y$ and the latent component, $\mathbf{w}$ is maximized when each of the latent components does not have any correlation.
Generally, the PLS only has one objective function. This objective function that is maximized on PLS for $i=1,2, \ldots, c$ will produce a weighting vector using (15)

$$
\mathbf{w}_{i}=\arg \max _{i} \mathbf{w}^{\mathrm{T}} \mathbf{X}^{\mathrm{T}} \mathbf{Y} \mathbf{Y}^{\mathrm{T}} \mathbf{X} \mathbf{w}
$$

as long as: $\mathbf{W}_{i}^{T} \mathbf{W}_{i}=1 ; \mathbf{w}_{i}^{T} \mathbf{X}^{T} \mathbf{X} \mathbf{W}_{j}=\mathbf{t}_{i}^{T} \mathbf{t}_{j}=0$, for $j=1,2, \ldots, i-1$.

We can see from the formula that the latent component formed on PLS has maximum covariance with the response variable so that the prediction is very good (Clark et al., 2001). PLS Algorithm (Boulesteix et al., 2006)

a. First iteration $h=1$, Maximum iteration

$$
h_{\max }=p
$$

b. Determine $\mathbf{w}=\mathbf{X}^{T} \mathbf{y} / \mathbf{y}^{T} \mathbf{y}$

c. Calculate $\mathbf{t}=\mathbf{X w}$

d. Calculate the loading $\mathbf{Y}, \mathbf{q}=\mathbf{y}^{T} \mathbf{t} /\left(\mathbf{t}^{T} \mathbf{t}\right)$

e. Renew $\mathbf{X}$ and $\mathbf{Y}$, as in (16)

$$
\begin{aligned}
& \mathbf{p}=\mathbf{X}^{T} \mathbf{t} /\left(\mathbf{t}^{T} \mathbf{t}\right) \\
& \mathbf{X}=\mathbf{X}-\mathbf{t p}^{T} \\
& \mathbf{Y}=\mathbf{Y}-\mathbf{t q}^{T}
\end{aligned}
$$

The value for measuring the goodness of the model's prediction is the determination of coefficient value $\left(R^{2}\right)$ that can be calculated using (17)

$$
\boldsymbol{R}^{2}=\mathbf{1}-\frac{\sum_{t=1}^{n}\left(y_{t}-\hat{y}_{t}\right)^{2}}{\sum_{t=1}^{n}\left(y_{t}-\bar{y}\right)^{2}}
$$

\subsection{Model Validation}

One of the measurements that can be used to know the quality of forecasting result is Root Mean Square Error of Prediction (RMSEP) (Wold et al., 2001). The formula we can use to obtain the RMSEP value from the univariate modeling is as (18).

$$
R M S E P=\sqrt{\frac{\sum_{t=1}^{n_{\text {pred }}}\left(Y_{t}-\hat{Y}_{t}\right)^{2}}{n_{\text {pred }}}}
$$

The smaller the RMSEP value, the better the forecasting model. The criteria of RMSEP value can be used as a base for model validation which is shown in Table 1. 
Table 1: RMSEP value criteria (Source: BMKG).

\begin{tabular}{ccc}
\hline \multirow{2}{*}{ Criterion } & \multicolumn{2}{c}{ RMSEP } \\
\cline { 2 - 3 } & Temperature & Humidity \\
\hline Very good & $0.0-0.4$ & $0.0-2.5$ \\
Good & $0.5-0.8$ & $2.6-5.0$ \\
Intermediate & $0.9-1.2$ & $5.1-7.5$ \\
Bad & $1.3-1.6$ & $7.6-10.00$ \\
Very bad & $>1.6$ & $>10.00$ \\
\hline
\end{tabular}

\subsection{Bias Corrector Measurement}

The percentage improvement of MOS model against the NWP is shown by the Percentage Improval (\% IM) that can be calculated using formulas as (19)

$$
\% I M=\frac{R M S E P_{N W P}-R M S E P_{M O S}}{R M S E P_{N W P}} \times 100 \%
$$

The value of $\%$ IM is from $0 \%$ to $100 \%$. The higher value of \%IM means the MOS model has a better correction of the NWP's biased forecasting result.

\subsection{Data and Variables}

The data used in this study is a secondary data from BMKG, i.e. the output of the daily NWP CCAM from 1 January 2009 to 31 December 2010. Four observation stations that are used in this study are Citeko, Kemayoran, Pondok Bentung, and Tangerang. The response variable is the surface's weather observation data that consist of Tmax, Tmin, and RH measured directly in every station. The predictor variable is the output of the NWP CCAM model. Meanwhile, the NWP CCAM parameter used is taken from the previous study's parameter by a meteorologist, shown in Table 2 for the MOS model.

The used parameters from the NWP CCAM for every observation station are 18 parameters. The 11 parameters are measured on the surface level (with a height of \pm 2 meters above the sea level), while the other 7 parameters are measured on a three level of different air pressures, where level 1 is 100 millibar pressure, level 2 is 950 millibar pressure, and level 4 is 850 millibar pressure. Therefore, the total parameters are 32 parameters. Each parameter is measured on 9 measurement grids $(3 \times 3)$ in the nearest location from the place of observation station. 
Table 2: NWP CCAM parameters.

\begin{tabular}{clc}
\hline No. & \multicolumn{1}{c}{ Variable } & Level \\
\hline 1 & Surface Pressure Tendency dpsdt) & Surface \\
2 & Water Mixing Ratio (mix) & $1,2,4$ \\
3 & Vertical Velocity (omega) & $1,2,4$ \\
4 & PBL depth (pblh) & Surface \\
5 & Surface Pressure (ps) & Surface \\
6 & Mean Sea Level Pressure (psl) & Surface \\
7 & Screen Mixing Ratio (qgscm) & Surface \\
8 & Relative Humidity (rh) & $1,2,4$ \\
9 & Precipitation (rnd) & Surface \\
10 & Temperature & $1,2,4$ \\
11 & Maximum Screen Temperature (tmaxcr) & Surface \\
12 & Minimum Screen Temperature (tmincr) & Surface \\
13 & Pan Temperature (tpan) & Surface \\
14 & Screen Temperature (tscrn) & Surface \\
15 & Zonal Wind (u) & $1,2,4$ \\
16 & Friction Velocity (ustar) & Surface \\
17 & Meridional Wind (v) & $1,2,4$ \\
18 & Geopotential Height (zg) & $1,2,4$ \\
\hline
\end{tabular}

\section{RESULTS AND DISCUSSION}

The analysis and evaluation steps for Tangerang Station will be explained in detail, while the rest of the stations will be just a slight summary since the occurrence analysis steps are actually the same.

\subsection{Pre-Processing the NWP Data using PCA Method}

Each NWP variable is measured on 9 measurement grids. Hence, there are 162 ( $18 \times 9$ ) predictor variables will increase the complexity of the model. To solve it, this study used a dimensional reduction i.e. PCA. The amount of principal components is determined by choosing which have an eigenvalue larger than one. The principal component for the NWP variable in Tangerang Station is shown in Table 3.

Table 3: NWP variable's principal components in Tangerang station.

\begin{tabular}{cccccccc}
\hline Variable & PC & $\begin{array}{c}\text { Eigen } \\
\text { Value }\end{array}$ & Var. & Variable & PC & $\begin{array}{c}\text { Eigen } \\
\text { Value }\end{array}$ & Var. \\
\hline Dpsdt & 1 & 9.2904 & 99.9857 & temp2 & 1 & 8.3576 & 97.1532 \\
mixr1 & 1 & 8.7048 & 92.4047 & temp4 & 1 & 8.7006 & 99.0090 \\
mixr2 & 1 & 8.9565 & 96.2157 & Tmaxscr & 1 & 8.5420 & 98.0922 \\
$\vdots$ & $\vdots$ & $\vdots$ & $\vdots$ & $\vdots$ & $\vdots$ & $\vdots$ & $\vdots$ \\
temp1 & 1 & 8.4996 & 95.8768 & zg4 & 1 & 8.5784 & 97.5735 \\
\hline
\end{tabular}

Table 3 shows that in Tangerang station, each NWP variables produces 1 component, except for the $z g$ level 1 variable that is 3 components, and zg level 2 variable that is 2 components. Therefore, the total amount of the principal components that are 
formed in Tangerang Station is 35 components, 39 components in Citeko Station, 35 components in Kemayoran and Pondok Betung Station. The variability of NWP variables explained by the principal components varies from $92.40 \%$ until almost $100 \%$. The principal components will be used as the predictor variables on the MOS modeling using PLS.

\subsection{Prediction Modeling of Tmax, Tmin, and RH using PLS Method}

The first step of PLS modeling in Tangerang Station is to determine the optimum amount of component of each model using a cross validation.

Table 4: The amount of the optimal components in four stations.

\begin{tabular}{llll}
\hline Station & Variable & Amount of components & $\begin{array}{l}\text { Smallest } \\
\text { PRESS value }\end{array}$ \\
\hline \multirow{3}{*}{ Citeko } & Tmax & 11 & 0.7317 \\
& Tmin & 7 & 0.8627 \\
& RH & 29 & 0.7554 \\
\hline \multirow{3}{*}{ Kemayoran } & Tmax & 9 & 0.7035 \\
& Tmin & 6 & 0.8627 \\
\hline \multirow{3}{*}{ Pondok Betung } & RH & 6 & 0.8653 \\
\hline \multirow{3}{*}{ Tangerang } & Tmax & 22 & 0.7154 \\
& Tmin & 5 & 0.9079 \\
& RH & 5 & 0.9084 \\
\hline
\end{tabular}

On the cross-validation process, every iteration will produce a PRESS value. Model with the smallest PRESS value will be the model that holds the optimum amount of components. The optimal component from the PLS in the four stations is shown in Table 4.

The optimal amount of component in each station is then used for the predictive modeling process of Tmax, Tmin, and RH. The modeling process will be explained according to the steps of the PLS modeling which have been described previously.

\section{Calculating PLS Weighting in Tangerang Station}

The weighting matrix $(\mathbf{W})$ is obtained from a merge of an every weighting vector extracted according to the amount of optimal component that has already been determined before. The component of $\mathbf{W}$ matrix in Tangerang Station (i.e. Tmax, Tmin, and RH) is shown in Table 5 , Table 6 , and Table 7 respectively. 
Table 5: The weight value of $X$ used for Tmax of PLS modeling in Tangerang station.

\begin{tabular}{rlrrrr}
\hline Variable & w1 & w2 & w3 & $\ldots$ & w6 \\
\hline PC.dpsdt & 0.0417 & 0.1248 & -0.1882 & $\ldots$ & -0.2782 \\
PC.mixr1 & -0.0130 & 0.0309 & -0.2308 & $\ldots$ & -0.2094 \\
PC.mixr2 & 0.0714 & 0.1292 & -0.2660 & $\ldots$ & 0.0641 \\
$\quad \vdots$ & $\vdots$ & $\vdots$ & $\vdots$ & $\ddots$ & $\vdots$ \\
PC.zg4 & -0.2603 & 0.1695 & -0.0784 & $\ldots$ & -0.1689 \\
\hline
\end{tabular}

Table 6: The weight value of X used for Tmin of PLS modeling in Tangerang station.

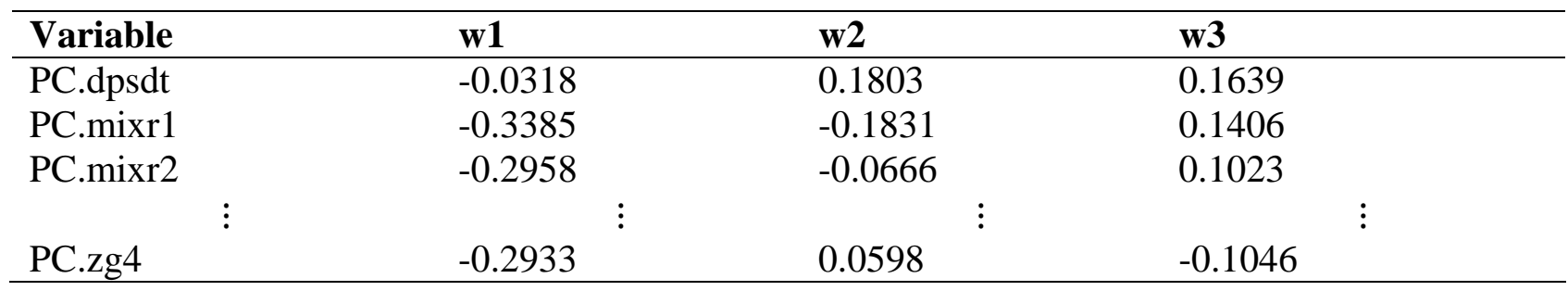

Table 7: The weight value of X used for RH of PLS modeling in Tangerang station.

\begin{tabular}{|c|c|c|c|}
\hline Variable & w1 & w2 & \\
\hline PC.dpsdt & -0.0651 & -0.0237 & \\
\hline PC.mixr1 & -0.1994 & -0.0207 & \\
\hline PC.mixr2 & -0.2408 & -0.0206 & \\
\hline PC.zg4 & 0.0579 & -0.3081 & $\vdots$ \\
\hline
\end{tabular}

\section{X-Scores Formation}

The obtained X-scores will be the $\mathbf{T}$ matrix consisted of a vector $\mathbf{t}$ component. The $\mathbf{X}$ matrix is the predictor matrix from the result of PCA operation, while $\mathbf{w}$ is the weighting value that is obtained previously. The $\mathrm{X}$-scores for Tmax is shown in Table 8, and Table 9 for the Tmin and $\mathrm{RH}$.

Table 8: The X-scores for Tmax of PLS modeling in Tangerang station.

\begin{tabular}{|c|c|c|c|c|}
\hline $\mathbf{N}$ & t1 & t2 & $\ldots$ & t6 \\
\hline 1 & -4.2611 & 0.1455 & $\ldots$ & -1.0065 \\
\hline 2 & -0.6483 & 1.0485 & $\ldots$ & -0.5370 \\
\hline 3 & -1.0081 & 2.1319 & $\ldots$ & -2.1069 \\
\hline & $\vdots$ & $\vdots$ & $\because$ & \\
\hline 637 & 0.9811 & -0.2294 & $\ldots$ & 0.2630 \\
\hline
\end{tabular}


Table 9: The X-scores for Tmin and RH of PLS modeling in Tangerang station.

\begin{tabular}{|c|c|c|c|c|c|}
\hline \multirow{2}{*}{$\mathbf{N}$} & \multicolumn{3}{|l|}{ Tmin } & \multicolumn{2}{|l|}{ RH } \\
\hline & t1 & t2 & t3 & t1 & t2 \\
\hline 1 & -1.1287 & 1.7879 & -1.7765 & 2.9974 & -2.8224 \\
\hline 2 & -1.4827 & 0.5584 & 1.5362 & -1.3023 & -2.7302 \\
\hline 3 & -3.9506 & 0.8837 & 3.6885 & -2.6981 & -3.8481 \\
\hline & $\vdots$ & $\vdots$ & & $\vdots$ & $\vdots$ \\
\hline 637 & 0.1494 & -0.9879 & -0.5842 & -0.5341 & 0.2010 \\
\hline
\end{tabular}

\section{Loading Factor Matrix Formation for $\mathrm{Y}$}

Y-loading is a loading related to the response variable. The loadings factor is obtained from the combination of loadings $\mathrm{Y}$ factor of each component. The loadings factor matrix for $\mathrm{Y}$ is shown in Table 10.

Table 10: Loadings Y factor of PLS modeling in Tangerang station.

\begin{tabular}{llllll}
\hline Q & Tmax & Q & Tmin & q & RH \\
\hline q1 & 0.2356 & q1 & 0.1354 & q1 & 0.2150 \\
q2 & 0.1921 & q2 & 0.0815 & q2 & 0.1577 \\
q3 & 0.1332 & q3 & 0.0532 & & \\
q4 & 0.0821 & & & & \\
q5 & 0.0874 & & & & \\
q6 & 0.0543 & & & & \\
\hline
\end{tabular}

4. Calculating Regression Coefficient

The PLS coefficient (B) can be obtained after matrix $\mathbf{W}, \mathbf{Q}$, and $\mathbf{T}$. The component of the PLS coefficient matrix on Tangerang Station is shown in Table 11.

Table 11: PLS coefficient in Tangerang station.

\begin{tabular}{|c|c|c|c|}
\hline Variable & Tmax & Tmin & RH \\
\hline PC.dpsdt & 0.0012 & 0.0243 & -0.0212 \\
\hline PC.mixr1 & -0.0320 & -0.0707 & -0.0566 \\
\hline PC.mixr2 & 0.0436 & -0.0520 & -0.0677 \\
\hline PC.zg4 & $\begin{array}{ll} & \vdots \\
-0.0553 & \end{array}$ & -0.0479 & -0.0331 \\
\hline
\end{tabular}

\section{PLS Preparation}

The preparation of PLS is done by the regression coefficient taken from Table 11 with the predictor variable that is obtained from PCA. When the PLS is formed, the conjectured value of the Tmax, Tmin, and RH can also be obtained. Those conjecture value, especially the conjectured value of the training data can be used to test how good the formed model with $R^{2}$ is, as the higher the $R^{2}$ value, the better the model is. The obtained $R^{2}$ value from the PLS in the four stations is shown in Table 12. 
Table 12: The value of $R^{2}$ from PLS in four stations.

\begin{tabular}{lll}
\hline Station & Variable & $\boldsymbol{R}^{\mathbf{2}(\boldsymbol{\%})}$ \\
\hline \multirow{3}{*}{ Citeko } & Tmax & 52.75 \\
& Tmin & 41.99 \\
& RH & 50.89 \\
\hline \multirow{2}{*}{ Kemayoran } & Tmax & 54.57 \\
& Tmin & 31.26 \\
\hline \multirow{2}{*}{ Pondok Betung } & RH & 46.33 \\
\hline \multirow{3}{*}{ Tangerang } & Tmax & 53.57 \\
& Tmin & 24.44 \\
& RH & 47.03 \\
\hline
\end{tabular}

Table 12 shows that the average value of $R^{2}$ obtained from the PLS is generally not that good, despite that the $R^{2}$ value for the Tmax modeling (maximum temperature as response) alone is good, ranging from $52.75 \%$ to $55.33 \%$, because the $R^{2}$ value from the Tmin modeling (minimum temperature as response) is quite small with a range of $14.93 \%-41.99 \%$ and the $R^{2}$ value from the $\mathrm{RH}$ modeling that ranges from $31.95 \%$ to $50.89 \%$. The $R^{2}$ value for the Tmax modeling in Tangerang Station is $55.33 \%$, means that there is $55.33 \%$ Tmax variance that can be explained by the formed model.

\subsection{PLS Validation}

The model validation aims to know the accuracy and the goodness of the formed model. The PLS validation is done by testing data with the observation data so that we can obtain the RMSEP value. The RMSEP value in four stations is shown in Table 13.

Generally, the RMSEP value of the Tmax modeling using PLS has an intermediate result according to the BMKG criterion. In the other side, the RMSEP value of the Tmin in Citeko, Kemayoran, and Pondok Betung Station has a good result of 1.0857. This PLS modeling is also has a good criterion if used for RH modeling in Citeko, Kemayoran, and Pondok Betung Station, while the RH modeling in Tangerang Station has an intermediate criterion because it holds the RMSEP value of 5.7314. The result of this PLS modeling is then regarded as the MOS model.

Table 13: RMSEP value for PLS in four stations.

\begin{tabular}{llll}
\hline Station & Variable & RMSEP & RMSEP Criterion \\
\hline \multirow{3}{*}{ Citeko } & Tmax & 1.1261 & Intermediate \\
& Tmin & 0.5183 & Good \\
& RH & 4.9307 & Good \\
\hline \multirow{3}{*}{ Kemayoran } & Tmax & 0.9698 & Intermediate \\
& Tmin & 0.7502 & Good \\
& RH & 4.3629 & Good \\
\hline \multirow{2}{*}{ Pondok Betung } & Tmax & 1.0479 & Intermediate \\
& Tmin & 0.8563 & Good \\
& RH & 4.6994 & Good \\
\hline
\end{tabular}




\begin{tabular}{llll} 
& Tmax & 0.9485 & Intermediate \\
Tangerang & Tmin & 1.0857 & Intermediate \\
& RH & 5.7314 & Intermediate \\
\hline
\end{tabular}

\subsection{Comparison of Accuracy between NWP Prediction Result and MOS Model Result}

The NWP model produces a biased forecast so that it needs a post-processing using the MOS method, i.e. PLS. The percentage of the amount of biased NWP data that can be corrected by the MOS model is shown by the
Percentage Improval (\%IM), where the RMSEPNWP is obtained based on the comparison of the NWP data on the fifth grid (the nearest grid from the observation station) and the observation data for the Tmax, Tmin, and $\mathrm{RH}$ variables. The amount of biased data that can be corrected by the MOS model with the PLS in the four stations are shown in Table 14.

Table 14: The value of RMSEP ${ }_{\mathrm{NWP}}$, RMSEP $\mathrm{ROS}_{\mathrm{MO}}$, dan \%IM.

\begin{tabular}{lllll}
\hline Station & Variable & RMSEPMOS & RMSEPNWP & \% IM \\
\hline \multirow{3}{*}{ Citeko } & Tmax & 1.1261 & 4.1572 & 72.9121 \\
& Tmin & 0.5183 & 5.1505 & 89.9369 \\
& RH & 4.9307 & 13.0509 & 62.2195 \\
\hline \multirow{3}{*}{ Kemayoran } & Tmax & 0.9698 & 2.9491 & 67.1154 \\
& Tmin & 0.7502 & 1.9110 & 60.7431 \\
& RH & 4.3629 & 7.1804 & 39.2388 \\
\hline \multirow{3}{*}{ Pondok Betung } & Tmax & 1.0479 & 3.3227 & 68.4624 \\
& Tmin & 0.8563 & 1.0812 & 20.8010 \\
& RH & 4.6994 & 7.5821 & 38.0198 \\
\hline \multirow{3}{*}{ Tangerang } & Tmax & 0.9485 & 3.1089 & 69.4908 \\
& Tmin & 1.0857 & 1.3400 & 18.9776 \\
& RH & 5.7314 & 6.5589 & 12.6164 \\
\hline
\end{tabular}

Table 14 shows that the RMSEP that is obtained from the NWP model is greater than the RMSEP from the MOS model, which means that the MOS model is consistently better to be used to predict the Tmax, Tmin, and $\mathrm{RH}$ rather than the NWP model. The MOS model is able to correct from $18.9776 \%$ to $89.9369 \%$ of the biased NWP for forecasting the Tmin. The same table also shows that the RMSEP $_{N W P}$ in the Citeko Station is the greatest among the other four stations so that Citeko Station has a \%IM that holds the greatest bias corrector. This is because the Citeko Station is located in the mountain area that holds a complex vegetation, therefore producing a big amount of bias for the NWP model.

\section{CONCLUSION}

Most of the principal components that are formed by the result of the NWP variables reduction within the 9 measurement grids are exactly one component. The validation result of the PLS with the RMSEP criterion shows that the Tmax belongs to intermediate for all stations, Tmin has a good criterion in three stations (i.e. Citeko, Kemayoran and Pondok Bentung), and RH has a good criterion in three stations (i.e. Citeko, Kemayoran and Pondok Bentung). The prediction results from the PLS is more accurate than the NWP model and able to correct an $89.94 \%$ of the biased NWP for Tmin forecasting (response as a result of PLS modeling). Therefore, we can conclude that the 
PLS can solve the NWP problem regarding the relation function and dimension reduction.

The modeling result from this study is recommended to be used for BMKG in forecasting the temperature and humidity because this model is capable to produce a smaller bias compared to the NWP model from the BMKG itself. It must be noted that a method comparison should be done in each station to obtain the best method due to a potential spatial effect that may occur.

\section{ACKNOWLEDGEMENT}

The entire data used in this study were supported by the Meteorology, Climatology and Geophysics Agency (BMKG) of Indonesia. The fund for this study is supported by the Ministry of Research, Technology and Higher Education of Indonesia for the grant of the National Strategic Research 2018.

\section{REFERENCES}

BMKG. (2006). Uji Operasional dan Validasi Model Output Statistik (MOS). Jakarta: BMKG.

Boulesteix, Anne-Laure, and Strimmer, K. (2006). Partial Least Squares: A Versatile Tool for the Analysis of High-Dimensional Genomic Data. Journal of Briefings in Bioinformatics, 8: 32-44.
Clark, M. P., Hay, L. E., and Whitaker, J. S. (2001). Development of operational hydrologic forecasting capabilities. American Geophysical Union, Fall Meeting.

Glahn, H. R., and Lowry, D. A. (1972). The Use of a Model Output Statistics (MOS) in Objective Weather Forecasting. Applied Meteorology, 1203-1211.

Johnson, R. A., and Winchern, D. W. (2007). Applied Multivariate Statistical Analysis $6^{\text {th }}$ Edition. United States: Pearson Education.

Joliffe, I. T. (1986). Principal Component Analysis (2nd ed.). New York: Springer-Verlag.

Tjasyono, B. (2004). Klimatologi $2^{\text {nd }}$ Edition. Bandung: ITB.

Wardani, I. K. (2010). Manfaat Prediksi Cuaca Jangka-Pendek Berdasarkan Data Radiosonde dan Numerical Weather Prediction (NWP) untuk Pertanian Daerah.

Wilks, D. S. (2006). Statistical Methods in the Atmospheric Sciences $2^{\text {nd }}$ Edition. Boston: Elvesier.

Wold, S., Sjostrom, M., and Eriksson, L. (2001). PLS-regression: a basic tool of chemometrics. Journal of Chemometrics and Intelligent Systems, 58: 109-130. 